\title{
MedienPädagogik
}

Zeitschrift für Theorie und Praxis der Medienbildung

\section{Leseförderung 4.0?}

\section{Gibt es Unterschiede in den Merkmalen effektiver Lesefördermassnahmen mit multiplen Dokumenten, wenn digitale Medien genutzt werden?}

\author{
Maik Philipp
}

\section{Zusammenfassung}

Das Lesen verlagert sich zunehmend in digitale Medien und basiert dabei auf multiplen, teils multimodalen Dokumenten. Eine zeitgemässe Leseförderung muss dies aufgreifen, da das digitale Lesen neue Herausforderungen mit sich bringt. Dies ist Anlass einer quantitativen Reanalyse 24 kriterienbasiert ausgewählter Interventionsstudien, von denen rund die Hälfte mit digitalen Medien operierte. Die leitende Frage war dabei, ob sich a) in den informatorischen Prozessen, b) den allgemeinen instruktionalen Merkmalen und c) in den verwendeten Übungsdokumenten Differenzen auftun. Für jede Studie wurden bis zu 46 Variablen kodiert, und diese Merkmale dann in Gruppenvergleichen geprüft. Die Anzahl signifikanter Unterschiede war mit nur sechs sehr gering und betraf personale Formen der Strategievermittlung sowie Art und Anzahl der Dokumente. 


\title{
Reading Instruction 4.0? Are There Any Differences in the Characteristics of Effective Instructional Approaches with Multiple Documents When Using Digital Media?
}

\begin{abstract}
Reading becomes increasingly a digital activity, which additionally is based on multiple, partly multimodal documents. An appropriate reading instruction must take these current changes into account, since digital reading gives rise to new challenges for readers. This is the reason for this quantitative reanalysis of 24 intervention studies, which were selected after a thorough application of criteria. Almost half of the studies used digital media, while the other half did not. The guiding research question was, whether there were any differences between these groups regarding a) informational processes, b) general instructional features and c) document types being used. For this purpose, 46 variables were coded and compared. The number of statistically significant differences was modest (six instances) and only evident within strategy instruction with human instructors and collaborators on the one side and the quantity and partly the quality of documents on the other side.
\end{abstract}

\section{Einleitung: Quo vadis, Lesen? Eine alte Kulturtechnik im (digitalen) Wandel}

Mit der fortschreitenden Digitalisierung ändert sich auch der Lesealltag, und damit verändert sich auch das, was unter kompetenten Lesen verstanden wird. Dies lässt sich prototypisch in der jüngsten PISA-Studie nachweisen: Die Testung erfolgte rein computerbasiert, das Konstrukt «Lesekompetenz» erstreckte sich auf Aufgaben zur Unterscheidung von Fakt und Meinung, und es wurden in einigen Aufgaben typischerweise nur im Internet vorkommende Texte wie Foren-Beiträge verschiedener Personen berücksichtigt (OECD 2019). Diese Veränderung im Konstrukt und in der Testung sind sozialen und technischen Veränderungen geschuldet, die am Lesealltag und seinen Anforderungen nicht spurlos vorübergegangen sind. Im Gegenteil: Sie verändern die lesebezogenen Anforderungen und damit auch das Soll an Lesefähigkeiten, um gemäss dem 
Reading-Literacy-Konzept von PISA ausreichend lesekompetent zu sein. Daraus ergibt sich die Frage, wie Personen auf diese veränderlichen und veränderten Erfordernisse adäquat in formellen Bildungskontexten vorbereitet werden können, in denen lesedidaktische Massnahmen auch mit digitalen Medien erfolgen könnten - und sollten.

Doch was sind die Veränderungen im Lesealltag, mit denen sich Personen konfrontiert sehen? Die Anzahl der verfügbaren Dokumente steigt, editoriale Qualitätssicherungsmassnahmen werden seltener, widersprüchliche und fragwürdige Quellen (Stichwort: Fake News) stehen neben zuverlässigen, und zusehends sind es die Leserinnen und Leser selbst, die selektionsbedingt Kohärenz zwischen Texten und Informationen zu stiften haben (Magliano et al. 2017). Das digitale Lesen braucht damit zusätzliche, andere und elaboriertere Strategien des Lesens, welche sich an die Eigenheiten des digitalen Lesens multipler Dokumente anpassen, aber auch noch Merkmale des Lesens analoger einzelner Texte aufweisen (Cho und Afflerbach 2017). Darunter fallen zunehmend auch evaluativ-reflexive Fähigkeiten, welche im medienpädagogischen Diskurs unter der Dimension der Medienkritik versammelt werden (Groeben 2002).

Die Leseforschung hat begonnen, den durch die Digitalisierung energisch vorangetriebenen Wandel des Lesens in theoretischen Arbeiten und empirischen Studien zu adressieren. Als ein derzeit besonders auffälliger, in Theorie und Empirie fruchtbarer und insgesamt prototypischer Zugang erweist sich das Lesen multipler digitaler Dokumente (Bråten, Braasch und Salmerón 2020; Leu et al. 2017; Magliano et al. 2017; Singer und Alexander 2017, 1024). Mit dem Ausdruck ist eine Trias gemeint:

- Multipel bedeutet, dass es nicht mehr nur darum geht, einen einzelnen Fliesstext zu rezipieren, sondern mindestens zwei. Multipel kann sich nicht nur auf die Quantität beziehen, sondern auch auf die Qualität der Zeichensysteme und Modalität. Inhalte können in multiplen Modalitäten - etwa in depiktionaler Form - vorliegen, was einen erweiterten Textbegriff impliziert.

- Digital heisst, dass sich die Materialität der Lesemedien verändert, das Lesen also auf dem Bildschirm stattfindet. Hinzu kommt, dass die elektronischen Medien es erlauben, dynamische Texte, Hyperlink-Strukturen und multimodale Dokumente zu rezipieren. 
- Dokumente schliesslich sind ein Konglomerat nicht nur von den Texten mit ihren Inhalten, sondern im Verbund mit quellenbezogenen (Meta-) Daten, also Daten über den Kontext des Textes und seine Verfasser - je nach Ausweitung auch über Stil und weitere Informationen über die Informationen. Dokumente beinhalten also inhaltstragende Informationen und potenziell inhaltlich relevante Zusatzinformationen auf einer anderen Ebene.

Mit diesem neuen Phänomen, das längst in der Fläche angekommen ist und das andere Anforderungen an Leserinnen und Leser bereithält (Magliano et al. 2017, 346-51), ergibt sich eine Aufgabe, die sich in der Schnittmenge von Lesedidaktik und Medienpädagogik verorten lässt. Denn in Zeiten von «Fake News» sowie ungesicherter Herkunft von Informationen bei gleichzeitig potenziell unendlich vielen verfügbaren Dokumenten im Internet stellt sich die Frage nach dem, was den Wesenskern von Lesekompetenz gegenwärtig aus theoretischer und empirischer Sicht ausmacht. Und es stellt sich die Frage danach, wie ein solch kompetentes Lesen angemessen zu fördern ist.

Hier setzt der vorliegende Beitrag an. Er widmet sich der kognitionspsychologisch grundierten Frage, ob sich aus dem emergierenden Feld der effektiven Leseförderung mit multiplen Dokumenten Eigenheiten extrahieren lassen, wenn digitale Medien verwendet werden. Denn obwohl der Einsatz digitaler Medien durchaus üblich ist (Barzilai, Zohar und Mor-Hagani 2018, 990), steht eine systematische Betrachtung noch aus. Dies ist das Hauptziel des Beitrags, nämlich innerhalb einer Reanalyse bereits vorgenommener Auswertungen (Philipp 2019b) die Eigenheiten von effektiven Lesefördermassnahmen mit und ohne Einsatz digitaler Medien zu prüfen. Mit digitalen Medien sind hier Bildschirmmedien gemeint, welche innerhalb der Förderung für das Finden und Rezipieren, zum Teil aber auch für die Informationsaufbereitung mithilfe spezifischer Software zum Einsatz kamen.

Der Beitrag hat folgenden Aufbau: Zunächst stehen theoriegeleitete Überlegungen an, mittels derer die Spezifika des Lesens multipler (digitaler) Dokumente besser abschätzbar werden (Abschnitt 2). Es folgen Beschreibungen der Methode (3) nebst den Ergebnissen zu drei erkenntnisleitenden Fragestellungen (4). Ein Fazit mit Hauptergebnissen und Ergebnisdiskussion rundet den Beitrag ab (5). 


\section{Theoretischer Hintergrund}

Das Lesen multipler (digitaler) Dokumente zieht viel wissenschaftliche Aufmerksamkeit auf sich. In diesem Abschnitt werden zwei komplementäre, kognitiv orientierte Perspektiven zur Thematik präsentiert. Zum einen geht es darum, welche beiden Hauptprozesse - das Integrieren und das Sourcing - dazu benötigt werden, eine idealtypische internale Repräsentation gelesener Dokumente - ein Dokumentenmodell - aufzubauen (2.1). Da dieses Modell noch unspezifisch hinsichtlich der Eigenheiten digitalen Lesens ist, wird es zum anderen von einigen schlaglichtartigen Perspektiven zum digitalen Lesen flankiert. Diese betreffen erhöhte Anforderungen an kognitive Ressourcen, (meta-)kognitive Strategien und den aktuell beobachtbaren Nachteil des Lesens am Bildschirm für das Leseverstehen (2.2).

\subsection{Multiple Dokumente verstehen}

Der verstehende Umgang mit multiplen Dokumenten ist in verschiedenen, vor allem kognitionspsychologisch grundierten Modellen konzeptualisiert und beschrieben worden (Philipp 2018b). Ein zentrales Referenzmodell ist das «Dokumentenmodell» (Perfetti, Rouet und Britt 1999), welches in Abbildung 1 dargestellt ist. Das Dokumentenmodell besteht aus zwei Ebenen mit miteinander verbundenen Komponenten, welche als kognitive Produkte möglichst vernetzt verbunden vorliegen sollen, um Sets multipler Dokumente zu verstehen. Diese Produkte des Verstehens bedürfen kognitiver Prozesse, welche als «Integrieren» und als «Sourcing» bezeichnet werden (Rouet und Britt 2014). Da diese beiden Prozesse - bzw. richtiger: Prozessgruppen - im Zentrum des Beitrags stehen, verdienen sie eine genauere Betrachtung (s. für eine ausführliche Darstellung Philipp 2019a, 40-148). Das Dokumentenmodell ist eine idealtypische Variante des Leseverstehens multipler Dokumente, das in seiner ganzen Fülle eine Vielzahl schwierigkeitsgenerierender Merkmale enthält und deshalb anfällig ist für Fehlleistungen (Philipp 2019a, 316-24). 


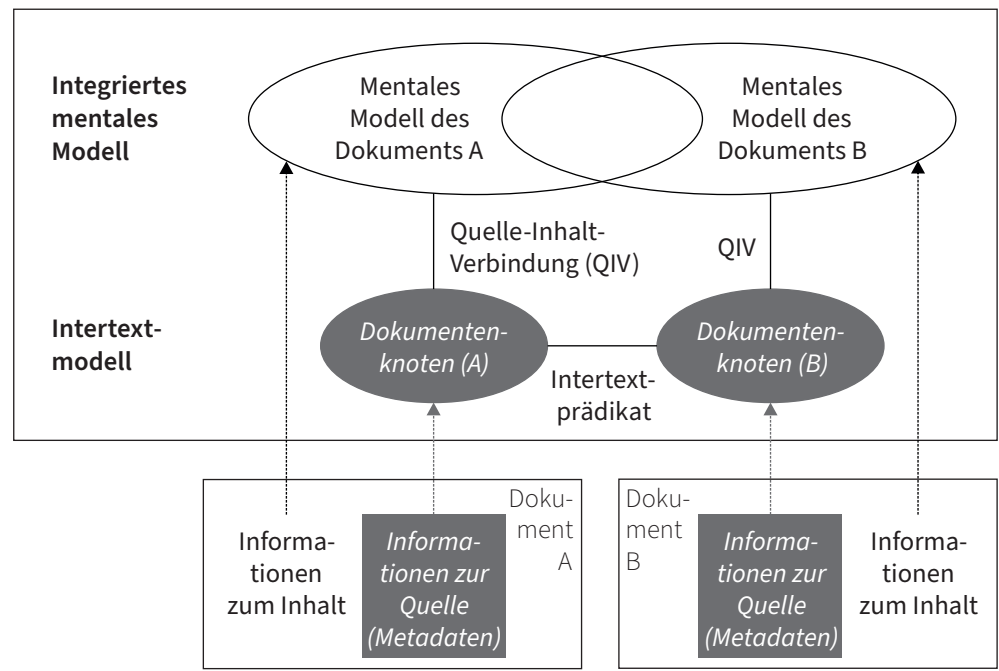

Abb. 1.: Komponenten des Dokumentenmodells mit besonderem Fokus auf Elemente, die für das Sourcing (grau und kursiv hervorgehoben) und für das Integrieren (ohne typografische Hervorhebung) von hoher Bedeutung sind (Quelle: Darstellung gemäss Britt und Rouet 2012, 285, entnommen aus Philipp 2019a, 36)

Das Integrieren - verstanden als Verknüpfen, Kombinieren und Organisieren von Informationen aus verschiedenen Dokumenten zu verschiedenen Zwecken (Barzilai et al. 2018, 976) - ist zentral für das Dokumentenmodell. Bei dem auf Inferenzleistungen basierenden Integrieren geht es darum, Informationen kohärent zusammenzuführen, und das galt bereits für einzelne Texte. Im Falle des Lesens multipler Dokumente braucht man das Integrieren dafür, für jedes einzelne gelesene Dokument ein mentales Modell des Inhalts zu konstruieren und insbesondere die inhaltlichen Schnittmengen und Bezüge über die Dokumentengrenzen hinweg zu erkennen. Dadurch erst können Personen ein «integriertes mentales Modell» auf der gleichnamigen Ebene im Dokumentenmodell entwickeln, welches eine komplexe Leistung im Sinne einer Verschmelzung von Vorwissen und diversen intra- und intertextuellen Dokumenteninhalten darstellt. Das Integrieren benötigt man zudem (auf der Ebene des Intertextmodells), um das intertextuelle Verhältnis von Dokumenten zu erkennen und zu benennen. Dies wird als «Intertextprädikat» bezeichnet und ist eine hochabstrakte Leistung der Leserin bzw. des Lesers. Eine letzte kognitive 
Integrationsleistung bildet im Dokumentenmodell die Verknüpfung von einzeldokumentbezogenen mentalen Modellen mit den sogenannten «Dokumentenknoten» als systematische Sammlung von Metadaten. Diese «Quelle-Inhalt-Verbindungen» genannten vertikalen Verknüpfungen verbinden die beiden Ebenen des integrierten mentalen Modells mit der des Intertextmodells.

Mit den «Dokumentenknoten» ist der im Dokumentenmodell aus Abbildung 1 typografisch hervorgehobene Bereich schon angesprochen, welcher für das Sourcing einschlägig ist. Das Sourcing bezeichnet als Sammelbegriff eine Gruppe von Prozessen, um vorhandene oder verfügbare Metadaten über Dokumente zu identifizieren, zu beurteilen, kognitiv zu repräsentieren und für die Relevanz- und Plausibilitätseinschätzung zu nutzen (Brante und Strømsø 2018, 777). Den Sammelort für Metadaten bilden also die Dokumentenknoten, welche bei der Einschätzung insbesondere bei inhaltlich konfligierenden Dokumenten von hohem Wert sind, weil sie das Zustandekommen von Dissens bzw. von Kohärenzproblemen besser erklären, ihrerseits aber eine hohe Kompetenz bei der Einschätzung und Nutzung von Metadaten voraussetzen. Hierin liegt eine hohe Anschlussfähigkeit an die Teildimension der Medienkritik/medienbezogenen Kritikfähigkeit als Teil des übergeordneten Konstrukts Medienkompetenz (Groeben 2002, 172-4).

\subsection{Spezifika digitalen Lesens}

Die Besonderheiten des digitalen Lesens wurden in der Vergangenheit vor allem dateninduziert rubriziert und zusammengefasst, wobei das analoge Lesen immer wieder den Hintergrund bildete (Cho und Afflerbach 2017; Salmerón et al. 2018; Wylie et al. 2018). Die Spezifika aus der kognitiven Sicht ganz allgemein haben Wylie et al. (2018) entlang vierer zusammenhängender kognitiver Themen beschrieben:

- Durch ihre typografischen Gestaltungen wie Unterstreichungen (z.B. auch bei Links) erfordern digitale (Hyper)Texte erstens gesonderte Aufmerksamkeit - diese Qualität tritt insbesondere bei Hypertexten auf. 
- Zweitens müssen Personen beim Lesen digitaler (Hyper-)Texte viele und verschiedenartige Informationen verarbeiten, was hohe Anforderungen an die Nutzung des Arbeitsgedächtnisses stellt.

- Drittens und mit beidem Genannten zusammenhängend müssen Leserinnen und Leser störende Impulse unterdrücken und stark zielgerichtet vorgehen, also ihre exekutiven Funktionen effektiv nutzen.

- Dazu zählt - viertens und letztens - die stark selbstregulative, metakognitive Steuerung des eigenen Lesens.

Insgesamt deuten sich damit hohe Anforderungen an Leserinnen und Leser an, wenn sie beim digitalen Lesen ihre notwendigen kognitiven Ressourcen optimal und gezielt nutzen und orchestrieren.

Dies leitet über zu einem weiteren Bereich, der als zentral gilt: die Lesestrategien, verstanden als kognitive und metakognitive Handlungen und Handlungspläne, um das Verstehen von Dokumenten zu erreichen. Das Lesen multipler Dokumente gilt als hochgradig lesestrategisch (List 2020), und hierbei gibt es Strategien in verschiedenen Bereichen:

- das Finden von Dokumenten im Internet, also ein gezieltes Recherchieren nach geeigneten Informationen und Dokumenten (Cho und Afflerbach 2017, 125; Cho, Afflerbach und Han 2018, 138; Salmerón et al. 2018, 93-5),

- das Herstellen von intertextuellen Bezügen zwischen den Dokumenten (Cho und Afflerbach 2017, 116-21, Salmerón et al. 2018, 98-100),

- das (sourcingbasierte) Evaluieren der digitalen Dokumente (Cho und Afflerbach 2017, 116-21; Cho et al. 2018, 137f.; Salmerón et al. 2018, 1024) sowie

- das gezielte und überwachte Navigieren zwischen Dokumenten (Cho und Afflerbach 2017, 121-8; Cho et al. 2018, 138).

Diese Strategien des Findens, Verknüpfens und Beurteilens von Informationen und Dokumenten stellt neue Anforderungen an Leserinnen und Leser, die teils quer zu den oben angeführten Bereichen der Aufmerksamkeit, Arbeitsgedächtnisnutzung, des Einsatzes der exekutiven Funktionen und Selbstregulation liegen. Vollumfänglich betrachtet bilden die lesestrategischen Anforderungen das Zentrum des Leseverstehens und Nutzens digitaler multipler Dokumente, darunter Hypertexte. 
Ein letzter, weniger theoretischer, dafür theoriebedürftiger Punkt ist erst in jüngerer Vergangenheit deutlich in der Forschung zum digitalen Lesen erkennbar geworden: der Mediumseffekt bzw. Bildschirmunterlegenheitseffekt. Damit ist gemeint, dass es anscheinend systematische Effekte des Präsentationsmediums von Texten auf das Leseverstehen gibt. Dieser Effekt ist auf breiter empirischer, nämlich inzwischen metaanalytischer Basis verschiedentlich nachgewiesen worden (Clinton 2019, 306; Delgado et al. 2018, 30f.; Kong, Seo und Zhai 2018, 143): Personen weisen schlechtere Verstehensleistungen bei digitalen (singulären) Texten im Vergleich zum Lesen analoger Texte auf. Einen besonders auffälligen Effekt förderten dabei Delgado et al. $(2018,32)$ zutage: Der Präsentationseffekt liess sich ausschliesslich dann nachweisen, wenn es sich bei dem Lesematerial entweder ausschliesslich um Sachtexte handelte oder Sachtexte Teil der gelesenen Texte waren. Der Nachteil digitalen Lesens besteht damit bei einem Textgenre, welches sowohl in der Grundlagenforschung (Primor und Katzir 2018, 5) als auch in der Förderung des Lesens multipler Dokumente (Barzilai et al. 2018, 990; Philipp 2019b, 15f.) absolut dominiert.

\subsection{Zwischenfazit}

Die bisherigen Ausführungen haben gezeigt, dass das Lesen multipler Dokumente ein kognitiv anspruchsvoller Vorgang ist, der diverse Prozesse der Informationsverknüpfung und -einschätzung erfordert. In Bezug auf das digitale Lesen scheinen die kognitiven Anforderungen nochmals höher zu sein. Wenn die Anforderungen beim Lesen digitaler (multipler) Dokumente hoch sind, stellt sich die Frage, ob diesen Eigenheiten auch in Fördermassnahmen Rechnung getragen wird. Insbesondere ist unklar, ob sich bei Fördermassnahmen mit digitalen Medien als Teil des Fördersettings Spezifika benennen lassen. Dies bildet den Anlass der vorliegenden Reanalyse von Studien, die sich zunächst einmal ganz explorativ begreifen lässt: Es geht nicht um die Überprüfung theoretischer, gerichteter Hypothesen. Vielmehr ist der Anlass des Beitrags, Gemeinsamkeiten und Unterschiede zwischen effektiven Fördermassnahmen mit und ohne Einsatz digitaler Medien zu eruieren. Dies ist dem zwar zunehmenden Interesse an der Effektivität von Lesefördermassnahmen mit computerbasierter 
Unterstützung geschuldet (Cheung und Slavin 2013; Swart et al. 2019; Xu et al. 2019), allerdings lässt sich derzeit noch wenig absehen, welchen Mehrwert digitale Medien für die Leseförderung im Segment des Lesens multipler Dokumente haben.

Die allgemeine Inkonsistenz bei den Erträgen der Zuhilfenahme von digitalen Medien zur Verbesserung des Leseverstehens und anderer kognitiver Prozesse und Produkte des Lesens - so die hier vorgeschlagene Arbeitsdefinition von «Leseförderung 4.0» - wirft allgemein viele Fragen auf. So stellt sich die Frage, ob allgemeine und eher technische Merkmale digitaler Medien - etwa die Art der Lesemedien (Handgeräte vs. stationäre Geräte, Bildschirmgrössen, Grad an Interaktivität der digitalen Dokumente etc.) - systematisch mit potenziellen oder empirisch eingeholten Erträgen im Zusammenhang stehen, wofür es bislang wenig metaanalytisch grundierte Hinweise gibt (Delgado et al. 2018). Auch die inhaltlichen Merkmale - nämlich, welche Art von Kognitionen mit welchen Dokumenten und welcher Art von lesedidaktischer Inszenierung und Zielsetzung bei welchen Personengruppen zu Veränderungen im Leseverstehen führen - spielen selbstredend eine bedeutende Rolle, die ihrerseits mit qualitativen Analysen genauer zu bestimmen sind.

Dieser Beitrag nimmt hier eine Zwischenstellung ein, indem er die instruktionalen Merkmale möglichst mehrdimensional beleuchtet, dies allerdings in Bezug auf ein Korpus mehrerer Studien. Es ist nicht sein Ziel, tiefergehend den Aufbau und die Prinzipien von Leserfördermassnahmen zu analysieren, die sich der Förderung zweier zentraler kognitiver Prozesse des Lesens multipler Dokumente (nämlich dem Integrieren und dem Sourcing) verschrieben haben und dabei mit oder ohne digitale Medien operierten (s. dazu Philipp, 2018a, 258-65; 2019a, 310-4). Vielmehr geht es um eine Gesamtschau auf mehr als 40 Merkmale, die sich in allen berücksichtigten Studien kodieren liessen und anhand derer sich - zugegeben: grobe - Profile dieser Förderansätze als Gruppe bilden lassen. Diese Merkmale werdenin analog zum Vorgehen bei Barzilai et al. (2018) gezielt isoliert betrachtet. 


\section{Methode}

\subsection{Fragestellungen}

Trotz an Fahrt aufnehmender Forschungsaktivität und trotz der sich abzeichnenden Spezifika digitalen Lesens ist immer noch unterbelichtet, ob die Leseförderung mit multiplen Dokumenten die Besonderheiten digitalen Lesens aufnimmt, indem sich Unterschiede je nach Einsatz von digitalen Medien in der eigentlichen Förderung zeigen. Im Zentrum dieses Beitrags stehen deshalb drei eigene Fragestellungen, die alle mit den etwaigen Eigenheiten der Leseförderung mit und ohne Einsatz digitaler Medien korrespondieren. Dies sind die Fragestellungen:

1. Welche Besonderheiten bestehen bei der Förderung von Sourcing- bzw. Integrationsprozessen mit und ohne Einsatz digitaler Medien?

2. Gibt es Differenzen in den allgemeinen instruktionalen Merkmalen der Fördermassnahmen?

3. Welche Eigenheiten liegen bei den eingesetzten Dokumenten als Trainingsmaterial vor?

\subsection{Datengrundlage}

Die Datengrundlage dieses Beitrags bilden 24 Primärstudien, in denen insgesamt 3.563 Personen aus der Primarstufe (ab Klassenstufe 4) bis hin in die Tertiärbildung in quasiexperimentellen Settings im Umgang mit multiplen Dokumenten gefördert wurden. Diese Auswahl aus Studien kam zustande, indem gezielt solche Studien aus zwei systematischen Reviews (Barzilai et al. 2018; Brante und Strømsø 2018) erneut kodiert wurden, in denen mit multiplen Dokumenten als Trainingsmaterial die Fähigkeiten im Integrieren und/oder Sourcing gefördert wurden und die in einer der abhängigen, im Gesamt inhaltlich hochgradig heterogenen Variablen hohe, signifikante Effekte von $g \geq .40$ erbrachten (Philipp 2019b, 7f.). Die Ergebnisse dieser Sekundäranalyse wurden anderweitig vorgestellt und diskutiert (Philipp 2019b), doch eine spezifische und auf statische Signifikanz hin stattfindende Auswertung der Effekte des Einsatzes digitaler Medien in den Förderansätzen ist bislang nicht erfolgt. 
Den Datenpool für diese Analysen bilden die zwei Dutzend Studien, und die genauen Kodierungen der Studienmerkmale und das Vorgehen der Kodierung sind anderweitig in einer Open-Access-Publikation nachlesbar (Philipp 2019b, 8-10, 25-7). Für die Belange dieses Kapitels ist entscheidend, dass in 11 der Studien digitale Medien innerhalb der Fördermassnahmen Verwendung gefunden haben und in 13 der Studien nicht. Somit verteilen sich die Studien mit und ohne Einsatz digitaler Medien ungefähr paritätisch.

Inhaltlich weisen die Studien in puncto Medieneinsatz eine hohe Heterogenität auf, sei es in Studien mit, sei es in Studien ohne digitale Medien. In der letztgenannten Gruppe war es durchgängig üblich, dass die Lernmaterialien in den Fördermassnahmen direkt am Bildschirm rezipiert wurden. Zusätzliche, das Potenzial digitaler Medien ausnutzende Varianten einer Prozessunterstützung der Kognitionen kamen nur marginal zum Einsatz: Lediglich vier Studien verwendeten Annotationssysteme, am Bildschirm dargebotene Hinweise und Gamification-Elemente (Philipp 2019b, 17, 25).

\subsection{Kodierte Merkmale}

Die in diesem Beitrag präsentierten Befunde stellen nur einen Ausschnitt dar. In aller Regel wurden die 24 Studien binär kodiert, nämlich hinsichtlich des Vorkommens einzelner Merkmale (vorhandenen bzw. nicht vorhanden). Diese vorhandenen Merkmale bilden zugleich die abhängige Variable in den quantitativen Analysen, nämlich in Abhängigkeit des Vorkommens innerhalb von Studien mit und ohne Einsatz digitaler Medien. Die kodierten Merkmale sind knapp zusammengefasst in Tabelle 1.

I) Integrationsprozesse (Fragestellung 1)

- 1) Textuelle Informationen: $\left.{ }^{\circ} 1 a\right)$ Finden relevanter, also benötigter Informationen, ${ }^{+} 1$ b) Integrieren intratextueller Informationen (innerhalb einzelner Dokumente), 1c) Integrieren intertextueller Informationen (zwischen einzelnen Dokumenten), 1d) Intertextprädikate (Verhältnis von Dokumenten untereinander inferieren)

- 2) Integrieren von Informationen und Metadaten: $\left.{ }^{\circ} 2 a\right)$ Verbinden von Metadaten und den Aussagen der Quellen einzelner Dokumente, 2b) Verbinden von Metadaten und den Aussagen der Quellen multipler Dokumente 


\section{II) Sourcingprozesse (Fragestellung 1)}

- 1) Merkmale der Verfasserinnen und Verfasser: ${ }^{\circ}$ 1a) Person (Name), 1b) Merkmale/Position der Person, 1c) Zuverlässigkeit/Expertise der Verfasserinnen und Verfasser, 1d) Motivation/Ziel der Verfasserinnen und Verfasser sowie 1e) anderes (restliche Merkmale)

- 2) Merkmale der Quelle: $\left.{ }^{\circ} 2 a\right)$ Erscheinungskontext (Quelle, Ort, Zeit, Medium), 2b) Zuverlässigkeit der Quelle, 2c) Textsorte/Dokumententyp/Art der Quelle, 2d) anderes (restliche Merkmale)

- 3) Informationen (keine Sammelkategorie): 3a) Zuverlässigkeit der Informationen selbst, $\left.{ }^{+} 3 b\right)$ andere Merkmale der Informationsbeurteilung ${ }^{+}$

III) Allgemeine instruktionale Merkmale (Fragestellung 2)

- Wissen zur Textstruktur/-organisation (Wissen über prototypische inhaltliche Informationsanordnungen)

- Metakognitive Prozesse (Überprüfen der kognitiven Sourcing- und Integrationsprozesse)

- Wert des Sourcings/Integrierens (Bedeutsamkeit und Notwendigkeit des Sourcings/des Integrierens)

- Kriterien des Sourcings/Integrierens (Qualitäten des geglückten Sourcings/der geglückten Integration)

- Prozedurale Hinweise (Fragen bzw. Aufforderung, bestimmte Aktivitäten oder Schritte auszuführen)

- Annotation/Zusammenfassen (Notizen, Hervorhebungen bzw. Zusammenfassungen einzelner Dokumente)

- Graphic Organizers (grafische Repräsentationen wie Tabellen, Karten, Diagramme etc.)

- Schreiben (Verwenden schriftlicher Aufgaben zur Weiterverarbeitung gelesener Dokumente)

- Explizite Vermittlung (sichtbare und klar zum Ausdruck gebrachte, informationsreiche Instruktion)

- Modellieren (verbale, didaktisierte Demonstration der Sourcing- bzw. Integrationsprozesse)

- Kooperation (Dyaden oder Kleingruppen lösen gemeinsam Aufgaben)

- Einzelarbeit (individuelles Üben)

IV) Verwendete Dokumente als Lernmaterialien (Fragestellung 3) 
- Anzahl der verwendeten Dokumente: höchste Anzahl der Dokumente in einem Set von Trainingsdokumenten

- Dokumentenarten: 1) literarische Texte (fiktionale Texte, z. B. Geschichten oder Romane), 2) Primärtexte (Dokumente aus erster Hand wie Reden oder Briefe), 3) Sekundärtexte ${ }^{\circ}$ (nicht-fiktionale Texte zu Sachverhalten) - darunter noch subkodiert: 3a) Schulbuchauszüge, 3b) ExpertInnen-Texte (z. B. Fachtexte von ForscherInnen), 3c) Zeitungen/News, 3d) Websites, 3e) Sonstiges, 3f) statisches visuelles Material (Fotos, Zeichnungen), 3g) dynamisches visuelles Material (Videos, Animationen)

Tab. 1.: Kodierte Merkmale in den Studien (Quelle: Philipp 2019b, 9f.; Legende: - Sammelkategorie, für die das Vorhandensein von Ausprägungen der Unterkategorien gebündelt gezählt wurden; ${ }^{+}$Befund wurde in der Original-Sekundäranalyse nicht berichtet)

\subsection{Auswertungsmethode und -strategie}

Die Kodierungen der zwei Dutzend Studien werden im Ergebnisteil in Form von Balkendiagrammen zusammengefasst. Neben der prozentualen Angabe von kodierten Merkmalen im Sinne des Vorkommens in den Studien wird der Mittelwert der Anzahl verwendeter Dokumente im Training berichtet. Diese Ergebnisdarstellung erfolgt zunächst deskriptiv. In den Diagrammen werden die Befunde jeweils für alle Studien sowie gesondert für Studien mit und ohne digitale Medien ausgewiesen. Die Grundgesamtheit bilden damit entweder alle Studien oder alle Studien, in denen digitale Medien verwendet wurden oder nicht.

Neben diesen deskriptiven Ergebnissen wurden Chi-Quadrat-Tests durchgeführt, um die Häufigkeitsverteilungen in den Studien mit und ohne digitale Medien zu überprüfen. Bei der Anzahl verwendeter Dokumente als Übungsmaterialien wurde ein t-Test für unabhängige Stichproben berechnet. Berichtet werden Differenzen nur dann, wenn die Irrtumswahrscheinlichkeit $p<.05$ beträgt; eine Bonferroni-Korrektur erfolgte nicht. Zusätzlich zur Überprüfung auf statistische Signifikanz werden in den Diagrammen noch jene Kategorien mit kursiver Hervorhebung gekennzeichnet, in denen es zu einer Differenz von mindestens 20 Prozentpunkten kam. 


\section{Ergebnisse}

\subsection{Vorgängige Analysen}

Für den Pool der Studien wurde kodiert, worauf die Fördermassnahmen fokussierten, nämlich ob die Zielgrössen Integrationsprozesse, Sourcingprozesse oder eine Kombination aus beidem bildeten. Es gab also drei Gruppen von Studien: Studien mit alleinigem Fokus auf Sourcing (5 Studien), auf Integration (6 Studien) oder mit einer kombinierten Förderung beider Prozesse (13 Studien). Um systematischen Verzerrungen des Einsatzes von digitalen Medien zu prüfen, wurde ein erster Chi-Quadrat-Test durchgeführt. Dieser ergab, dass in keiner der drei Gruppen von Zielgrössen überzufällige Differenzen im Einsatz digitaler Medien gab $\left(X^{2}(2, N=24)=3.103\right.$, $p=.22 ; \mathrm{V}=.35$ ).

Ein weiterer Chi-Quadrat-Test wurde genutzt, um Alterseffekte zu prüfen. In der ursprünglichen Sekundäranalyse wurden die Altersgruppen nach höherer Primarstufe (3 Studien), unterer Sekundarstufe (8 Studien), höherer Sekundarstufe (6 Studien) und Tertiärbildung (7 Studien) kodiert. Der Test ergab auch hier keine Auffälligkeiten $\left(X^{2}(3, N=24)=4.148, p=.25\right.$; $\mathrm{V}=.43)$.

Somit ergibt sich aus diesen beiden vorgängigen Analysen nicht die Notwendigkeit, Alter und instruktionalen Fokus auf die kognitiven Prozesse gesondert in den weiteren Analysen zu betrachten. Im Pool der Primärstudien liessen sich damit keine Hinweise auf die Dominanz von digitalen Medien bei den anvisierten kognitiven Prozessen und in den Altersgruppen belegen.

\subsection{Förderung der Integrationsprozesse (Fragestellung 1)}

Im Bereich der Förderung der kognitiven Integrationsprozesse, deren Bezugspunkte in Abbildung 2 dargestellt sind, ergaben sich keine statistisch signifikanten Differenzen. Im Bereich der Verknüpfung von textuellen Informationen, die ein Schwergewicht bildeten, fokussierten Förderansätze mit digitalen Medien mehr das Finden von relevanten Informationen und 
das Bilden von Intertextprädikaten. Demgegenüber legten Förderansätze ohne digitalen Medieneinsatz einen Schwerpunkt auf das Verknüpfen von intra- und - noch deutlicher - von intertextuellen Informationen.

1) Textuelle Informationen

1a) Relevante Informationen finden

1b) Intratextuelle Informationen

1c) Intertextuelle Informationen 1d) Intertextprädikate

2) Textinformationen mit Metadaten

2a) Einzelne Dokumente

2b) Multiple Dokumente



\section{$89 \%$}

$58 \%$

$32 \%$

$79 \%$

$26 \%$

\section{$26 \%$}

$21 \%$

$21 \%$

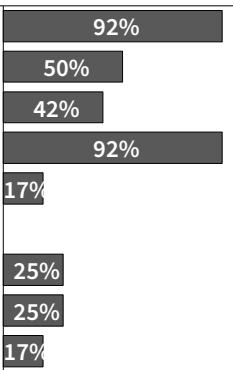

$17 \%$

- Gesamt

$\square$ Nutzung digitaler Medien $\quad \square$ keine Nutzung digitaler Medien

Abb. 2.: Prozentualer Anteil der Schwerpunkte beim Integrieren von Informationen, dargestellt für alle Studien und für solche mit bzw. ohne Nutzung digitaler Medien (Basis: 19 Studien (ohne Studien, in denen es nur um das Sourcing ging), gerundete Werte; Legende: Kursiv dargestellt sind Kategorien, in denen es eine Differenz von mindestens 20 Prozentpunkten in der Variante mit und ohne digitale Medien gab.)

\subsection{Förderung der Sourcingprozesse (Fragestellung 1)}

In den Sourcingprozessen unterschieden sich die Förderansätze ebenfalls nicht überzufällig (s. Abbildung 3). Gleichwohl legten die Fördermassnahmen mit digitalen Medien mehr Gewicht auf das Finden von Namen bei den Metadaten und insgesamt mehr auf quellenbezogene Metadaten. Demgegenüber waren die mit analogen Medien auskommenden Förderansätze stärker an erscheinungskontextuellen Metadaten und der informatorischen Zuverlässigkeit interessiert. 
1) Metadaten zu VerfasserInnen 1a) Name

1b) Merkmale/Position 1c) Zuverlässigkeit/Expertise 1d) Motivation/Ziel 1e) Anderes

2) Metadaten zur Quelle 2a) Erscheinungskontext

2b) Zuverlässigkeit 2c) Dokumentenart 2d) Anderes

3a) Informationen: Zuverlässigkeit 3b) Informationen: Anderes

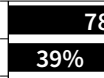

$28 \%$ $67 \%$ $50 \%$ $39 \%$ $8 \%$


$44 \%$ $39 \%$ $17 \%$ $28 \%$ $33 \%$ $22 \%$

\begin{tabular}{|c|c|}
\hline $78 \%$ & $78 \%$ \\
\hline $56 \%$ & $22 \%$ \\
\hline $22 \%$ & $33 \%$ \\
\hline $67 \%$ & $67 \%$ \\
\hline $44 \%$ & $56 \%$ \\
\hline $22 \%$ & $56 \%$ \\
\hline $78 \%$ & $56 \%$ \\
\hline $33 \%$ & $56 \%$ \\
\hline $33 \%$ & $44 \%$ \\
\hline $22 \%$ & $11 \%$ \\
\hline $33 \%$ & $22 \%$ \\
\hline $22 \%$ & $44 \%$ \\
\hline $33 \%$ & $11 \%$ \\
\hline
\end{tabular}

- Gesamt

$\square$ Nutzung digitaler Medien

Abb. 3.: Prozentualer Anteil der Schwerpunkte beim Sourcing, dargestellt für alle Studien und für solche mit bzw. ohne Nutzung digitaler Medien (Basis: 18 Studien (ohne Studien, in denen es nur um das Integrieren ging), gerundete Werte; Legende: Kursiv dargestellt sind Kategorien, in denen es eine Differenz von mindestens 20 Prozentpunkten in der Variante mit und ohne digitale Medien gab.)

\subsection{Allgemeine instruktionale Merkmale (Fragestellung 2)}

Hinter der Kategorie «allgemeine instruktionale Merkmale» verbergen sich diverse generische Merkmale, welche weniger mit den Sourcing- und Integrationsprozessen zu tun haben. Stattdessen handelt es sich in dieser Sammelkategorie um diverse rahmende instruktionale Elemente (s. Abbildung 4). Hierbei zeigten sich Tendenzen des stärkeren Einsatzes von der Vermittlung des Wissens über Textstrukturen und Textorganisationsformen und der Verwendung des Schreibens bei Studien ohne digitale Medien. Auch die Betonung des Werts und der Kriterien des Integrierens waren hier häufiger anzutreffen. Bei Studien mit digitalen Medien wurden metakognitive Prozesse stärker prononciert, und Graphic Organizers gelangten häufiger zum Einsatz.

Signifikante Differenzen mit stärkerer Ausprägung jeweils bei den Förderansätzen ohne digitale Medien bestanden bei der expliziten Vermittlung $\left(X^{2}(1, N=24)=6.331, p=.012 ; V=.51\right)$, der Verwendung des 
Modellierens $\left(X^{2}(1, N=24)=4.608, p=.032 ; V=.44\right)$ sowie der Kooperation $\left(X^{2}(1, N=24)=8.392, p=.004 ; V=.59\right)$. Diese Befunde lassen sich als stärkere Nutzung personeller Ressourcen umschreiben und bündeln.

\begin{tabular}{|c|c|c|c|}
\hline \multirow[b]{2}{*}{ Wissen Textstruktur/-organisation } & \multirow{2}{*}{\multicolumn{2}{|c|}{\begin{tabular}{l|l|}
$30 \%$ & $18 \%$ \\
\end{tabular}}} & \multirow{2}{*}{$42 \%$} \\
\hline & & & \\
\hline Metakognitive Prozesse & $52 \%$ & $73 \%$ & \\
\hline Wert des Sourcings* & $33 \%$ & $33 \%$ & $33 \%$ \\
\hline Kriterien des Sourcings ${ }^{\star}$ & $6 \%$ & $11 \%$ & $0 \%$ \\
\hline Wert des Integrierens ${ }^{\star *}$ & $21 \%$ & $0 \%$ & $33 \%$ \\
\hline Kriterien des Integrierens ${ }^{\star \star}$ & $21 \%$ & $0 \%$ & $33 \%$ \\
\hline Prozedurale Hinweise & $79 \%$ & $82 \%$ & $77 \%$ \\
\hline Annotation/Zusammenfassen & $58 \%$ & $55 \%$ & $62 \%$ \\
\hline Graphic Organizers & $54 \%$ & $73 \%$ & $38 \%$ \\
\hline Schreiben & $54 \%$ & $36 \%$ & $69 \%$ \\
\hline Explizite Vermittlung & $71 \%$ & $45 \%$ & $92 \%$ \\
\hline Modellieren & $42 \%$ & $18 \%$ & $62 \%$ \\
\hline Kooperation & $67 \%$ & $36 \%$ & $92 \%$ \\
\hline Einzelarbeit & $88 \%$ & $82 \%$ & $92 \%$ \\
\hline & $\begin{array}{l}\text { - Gesamt } \\
\square \text { Nutzung }\end{array}$ & dien & ung digitaler Medien \\
\hline
\end{tabular}

Abb. 4.: Prozentualer Anteil verschiedener instruktionaler Merkmale, dargestellt für alle Studien und für solche mit bzw. ohne Nutzung digitaler Medien (Basis: 24 Studien, * Basis: nur Studien mit Sourcing (18 Studien), ** Basis: nur Studien mit Integration (19 Studien), gerundete Werte; Legende: Kursiv dargestellt sind Kategorien, in denen es eine Differenz von mindestens 20 Prozentpunkten in der Variante mit und ohne digitale Medien gab. Fett und unterstrichen sind die Kategorien, in denen es statistisch signifikante Differenzen gab.)

\subsection{Verwendete Dokumente als Lernmaterialien (Fragestel- lung 3)}

Bei den verwendeten Dokumenten als Grundlage für die Förderung kognitiver Prozesse waren zwei Analysen leitend. Zum einen betraf dies die Anzahl der verwendeten Dokumente, zum anderen die Art dieser Dokumente. Zunächst zur Anzahl der Dokumente (s. Abbildung 5): In Studien, in denen digitale Medien verwendet wurden, lag der Mittelwert der beim Training zum Einsatz gekommenen Dokumente $(N=9, M=8.50, S D=4.41)$ erheblich 
höher als in den Studien ohne Einsatz digitaler Medien $(N=11, M=3.64$, $S D=1.91)$. Diese Differenz erwies sich als signifikant $(t(9)=-2,927, p=.017)$.

Anzahl Texte

\begin{tabular}{|c|c|c|}
\hline 5,7 & 8,5 & 3,6 \\
\hline$\square$ Gesamt & $\square$ Nutzung digitaler Medien $\quad \square$ keine Nutzung digitaler Medien \\
\hline
\end{tabular}

Abb. 5.: Mittelwert der eingesetzten Texte/Dokumente in den Fördermassnahmen, dargestellt für alle Studien und für solche mit bzw. ohne Nutzung digitaler Medien (Basis: 19 Studien mit expliziten Angaben zur Anzahl verwendeter Texte/Lernmaterialien; Legende: Fett und unterstrichen ist die Kategorie «Anzahl», in der es statistisch signifikante Differenzen gab.)

Bei der Art der eingesetzten Dokumente, die aus Abbildung 6 hervorgeht, lagen zwei bedeutsame Differenzen vor. Zum einen kamen in Studien ohne digitale Medien mehr Primärtexte zum Einsatz $\left(X^{2}(1, N=24)=5.371, p=\right.$ .020; $V=$.47). Zum anderen nutzten Studien mit digitalen Medien häufiger Websites $\left(X^{2}(1, N=24)=16.620, p<.001 ; V=.83\right)$. Ausserdem ergaben sich Tendenzen zur stärkeren Verwendung von Sekundärtexten in Studien mit digitalen Medien sowie von Texten von Fachexpertinnen und -experten in Studien ohne digitale Medien.

\begin{tabular}{|c|c|c|c|}
\hline 1) Literarische Texte & $4 \%$ & $0 \%$ & $8 \%$ \\
\hline 2) Primärtexte & $33 \%$ & $9 \%$ & $54 \%$ \\
\hline 3) Sekundärtexte & $88 \%$ & $100 \%$ & $77 \%$ \\
\hline 3a) Schulbuch & $13 \%$ & $9 \%$ & $15 \%$ \\
\hline 3b) ExpertInnen-Texte & $21 \%$ & $9 \%$ & $31 \%$ \\
\hline 3c) Zeitungen/News & $4 \%$ & $9 \%$ & $0 \%$ \\
\hline 3d) Websites & $46 \%$ & $91 \%$ & $8 \%$ \\
\hline 3e) Sonstiges & $17 \%$ & $0 \%$ & $31 \%$ \\
\hline 3f) Statisches visuelles Material & $4 \%$ & $0 \%$ & $8 \%$ \\
\hline \multirow[t]{2}{*}{ 3g) Dynamisches visuelles Material } & $0 \%$ & $0 \%$ & $0 \%$ \\
\hline & \multicolumn{2}{|l|}{$\begin{array}{l}\text { - Gesamt } \\
\square \text { Nutzung }\end{array}$} & zung \\
\hline
\end{tabular}

Abb. 6.: Prozentualer Anteil der eingesetzten Texte/Dokumente in drei verschiedenen (Unter-)Gruppen von Dokumenten, dargestellt für alle Studien und für solche mit bzw. ohne Nutzung digitaler Medien (Basis: 24 Studien, gerundete Werte; Legende: Kursiv dargestellt sind Kategorien, in denen es eine Differenz von mindestens 20 Prozentpunkten in der Variante mit und ohne digitale Medien gab. Fett und unterstrichen sind die Kategorien, in denen es statistisch signifikante Differenzen gab.) 


\section{Fazit}

\subsection{Mehr Gemeinsamkeiten als Unterschiede - die Essenz der Befunde}

Den Gegenstand des Beitrags bildeten effektive, lesestrategische Fördermassnahmen im Umgang mit multiplen Dokumenten, und das Erkenntnisinteresse fokussierte auf Gemeinsamkeiten und Differenzen dieser Fördermassnahmen, welche mit oder ohne digitale Medien innerhalb der Förderung operierten. Entlang dreier Gruppen von Vergleichsdimensionen den Bezugspunkten der beiden kognitiven Hauptprozesse Integrieren und Sourcing, den allgemeinen instruktionalen Merkmalen und dem Einsatz von Dokumenten - erfolgte eine quantitative Auswertung.

In dieser Sekundäranalyse wurden 46 Vergleiche in den Studien vorgenommen. Nur in sechs Vergleichen - also weniger als einem Siebtel - lagen tatsächlich statistisch überzufällige Differenzen vor (s. Tabelle 2). Das erste und vielleicht bereits wichtigste Ergebnis ist dabei: Bei den informatorischen Bezugspunkten sowohl im Sourcing als auch im Integrieren liessen sich statistisch überzufällige Differenzen nicht nachweisen. Hier gibt es demnach mehr Gemeinsamkeiten als Unterschiede. 


\begin{tabular}{|c|c|c|}
\hline $\begin{array}{l}\text { Unterscheidungsdimensi- } \\
\text { on (Fragestellung) }\end{array}$ & $\begin{array}{l}\text { Höherer Ausprägung bei } \\
\text { Fördervariante mit digi- } \\
\text { talen Medien }\end{array}$ & $\begin{array}{l}\text { Höherer Ausprägung } \\
\text { bei Fördervariante ohne } \\
\text { digitale Medien }\end{array}$ \\
\hline $\begin{array}{l}\text { Integrationsprozesse (Fra- } \\
\text { gestellung 1) }\end{array}$ & $\begin{array}{ll}- & \text { mehr Finden von rele- } \\
& \text { vanten Informationen } \\
- & \text { mehr Inferieren von } \\
& \text { Intertextprädikaten }\end{array}$ & 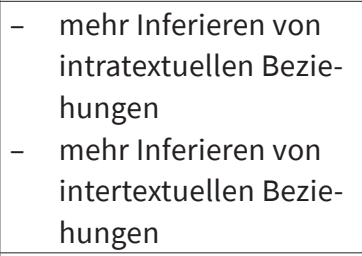 \\
\hline $\begin{array}{l}\text { Sourcingprozesse (Frage- } \\
\text { stellung 1) }\end{array}$ & $\begin{aligned}- & \text { mehr Finden von } \\
& \text { VerfasserInnen-Namen } \\
- & \text { mehr Finden von } \\
& \text { quellenbezogenen } \\
& \text { Metadaten } \\
- & \text { mehr sonstige Ein- } \\
& \text { schätzungen der Infor- } \\
& \text { mationen }\end{aligned}$ & 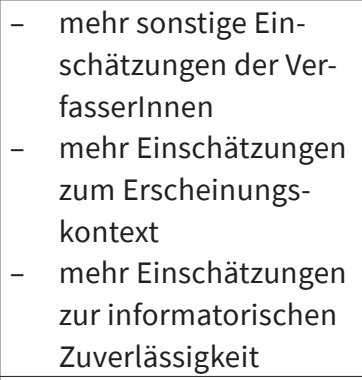 \\
\hline $\begin{array}{l}\text { Allgemeine instruktionale } \\
\text { Merkmale (Fragestellung } \\
\text { 2) }\end{array}$ & $\begin{aligned} \text { - } & \text { stärkerer Fokus auf } \\
& \text { Metakognition } \\
- & \text { mehr Nutzung von } \\
& \text { Graphic Organizers }\end{aligned}$ & 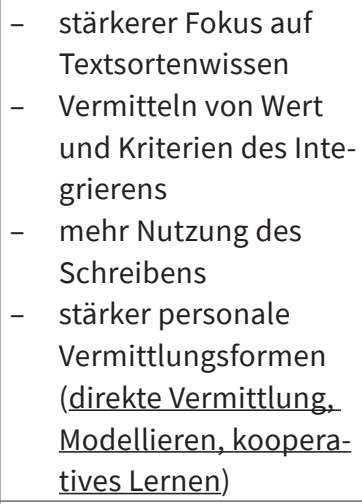 \\
\hline $\begin{array}{l}\text { Verwendete Dokumente } \\
\text { als Lernmaterialien (Fra- } \\
\text { gestellung 3) }\end{array}$ & $\begin{aligned}- & \text { mehr als doppelt so } \\
& \underline{\text { viele Dokumente ge- }} \\
& \underline{\text { nutzt }} \\
- & \text { mehr Sekundärtexte } \\
& \text { (v.a. Websites) genutzt }\end{aligned}$ & mehr Primärtexte genutzt \\
\hline
\end{tabular}

Tab. 2.: Hauptergebnisse des Vergleichs von Fördermassnahmen zum Integrieren und Sourcing bei multiplen Dokumenten (Legende: Unterstrichen sind die insgesamt sechs Kategorien, in denen statistisch signifikante Unterschiede beobachtbar waren, restliche aufgeführte Aspekte beziehen sich auf Kategorien, in denen es mindestens 20 Prozentpunkte Differenz gab.) 
Die sechs ermittelten signifikanten Unterschiede stammten paritätisch aus den allgemeinen instruktionalen Merkmalen und dem Einsatz der Dokumente zum Erwerb des lesestrategischen Vorgehens:

- Die Differenzen bei den instruktionalen Merkmalen betrafen allesamt Vergleichskategorien, die mit einem erhöhten Personalbedarf zu tun haben und die allesamt bei den Fördermassnahmen ohne digitale Medien häufiger vorkamen. Dies waren die explizite Vermittlung, das Modellieren des Vorgehens durch eine Modellperson und das kooperative Lösen von Aufgaben.

- Bei den eingesetzten Dokumenten als Lernmaterialien gab es drei Differenzen. In den Studien ohne digitale Medien dominierten Primärtexte deutlich, während bei den Fördermassnahmen unter Rückgriff auf digitale Medien Websites mehr und praktisch in jeder Studie Verwendung fanden. Ausserdem - und das ist ein ebenfalls markantes Resultat wurden in Studien mit digitalen Texten mehr als doppelt so viele Dokumente verwendet, nämlich mehr als acht, während es bei Studien ohne digitale Medien unter vier Dokumente waren.

Abgesehen von den Befunden mit statistischer Signifikanz gab es mehr als ein Dutzend Vergleiche, bei denen die Differenzen in den Studien mit und ohne digitale Medien mehr als 20 Prozentpunkte betrugen. Diese Befunde sind ebenfalls in Tabelle 2 dargestellt:

- Auffällig ist, dass mehr quellenbezogene und teils verfasserbezogene (hier: Namen) Metadaten bei Studien mit digitalen Medien geprüft wurden also in Studien, die praktisch ausnahmslos auf Websites setzten. Hier gibt es also eine Auffälligkeit in puncto Sourcing.

- Ebenfalls ein häufiger bei Studien mit digitalen Medien fokussierter Bereich sind die metakognitiven Prozesse, welche eine Optimierung des Integrierens und Sourcings ermöglichen. Dies nahmen drei von vier Studien auf.

- Schliesslich ist markant, dass bei den Studien mit digitalen Medien das Anordnen von Informationen in Graphic Organizers häufiger Bestandteil war, wofür man - auch dies eine Auffälligkeit in den Studien mit digitalen Medien - relevante Informationen suchen muss, die es grafisch zu organisieren gilt. Demgegenüber fand das Schreiben von Texten über Gelesenes in Studien ohne digitale Medien häufiger statt. 


\subsection{Abschliessende Diskussion der Resultate}

Das Lesen multipler digitaler Dokumente weist schwierigkeitsgenerierende Merkmale auf, die mit den beiden Hauptprozessen - dem Integrieren und dem Sourcing - korrespondieren. Das Lesen digitaler (singulärer) Texte hat zusätzliche schwierigkeitsgenerierende Eigenheiten, die auf einer erhöhten Beanspruchung kognitiver Ressourcen basieren und sich gerade bei Sachtexten in einem geringeren Leseverstehen manifestieren. Dies zeigt sich auch empirisch, denn eine Vielzahl von Studien hat demonstriert, dass Personen verschiedener Bildungsetappen hartnäckig von Schwierigkeiten im intertextuellen Integrieren und im Sourcing geplagt wird, die teils so gravierend sind, dass ein angemessenes Leseverstehen unwahrscheinlich wirkt (Philipp 2019a, 171-89 und 205-20).

Die vorliegende Sekundäranalyse von nachweislich effektiven Fördermassnahmen illustriert, dass einige der Spezifika digitalen Lesens bereits aufgegriffen werden. Daneben gibt es auch einige kritische Aspekte. Positiv hervorzuheben ist, dass mit der Nutzung digitaler Medien instruktionale Merkmale wie das grafische Organisieren und das metakognitive Überwachen unterstützt werden. Dies greift schwierigkeitsgenerierende Merkmale des intertextuellen Verknüpfens, aber auch des Verknüpfens von Inhalt und Metadaten auf, welche durch gesteuerte Sequenzen in Interfaces und das Einfordern von relevanten Informationen gezielt erleichtert werden können. Insgesamt stehen damit zentrale Strategien des Findens, Evaluierens und Verknüpfens von Informationen aus multiplen Dokumenten im Zentrum, auf die es beim Lesen multipler digitaler Dokumente ankommt (Cho und Afflerbach 2017; Salmerón et al. 2018).

Auffällig ist, dass Studien mit digitalen Medien eine andere Art von Förderung einschlagen, die mit weniger personalintensiven Merkmalen korrespondiert, während diese in Studien ohne digitale Medien deutlicher Bestandteil ist. Darunter fällt die explizite Vermittlung von Strategien mit Modellieren und kooperativen Elementen, die ihrerseits nicht nur klassisch für die Lesestrategievermittlung sind, sondern sich auch in diversen Studien mit multiplen Dokumenten wiederfinden (Philipp 2018a, 262-4). Die «kognitive Meisterlehre» geht (noch) von menschlichen Modellpersonen aus, die adaptiv ihre Fähigkeit explizit vermitteln und lernbezogene Scaffolds auf- und wieder abbauen. Gut möglich, dass in Zukunft von 
intelligenten tutoriellen Systemen ähnliche Effekte zu erwarten sind, wie sie schon in anderen, nicht-sprachlichen Domänen bestehen (Ma et al. 2014, 908f.; Swart et al. 2019; Steenbergen-Hu und Cooper 2014, 337). Gerade hier scheint sich auch anzudeuten, dass sich der mögliche Mehrwert digitaler Medien, etwa das automatisierte Feedback auf das Nutzerverhalten und die zeitnahen Feedbacks auf Leseleistungen innerhalb offener Aufgaben, in umfassender konzipierten Förderansätzen besser nutzen lässt (Magliano et al. 2018).

Eher kritischer Natur ist das Resultat, dass Fördermassnahmen mit digitalen Medien dazu neigen, viele Sachtexte zu nutzen. Dies mag ökologisch valide sein, jedoch liegen hierin zugleich schwierigkeitsgenerierende Merkmale, weil das Dokumentenset in solchen Settings ungleich komplexer wird, wenn es vollständig berücksichtigt wird, da mehr singuläre mentale Modelle zu integrierten mentalen Modellen verschmolzen werden müssen, mehr Dokumentenknoten mit mehr Metadaten zu füllen sind und mehr Quelle-Inhalt-Verbindungen und mehr Intertextprädikate zu generieren sind (s. O., Abschnitt 2.1). Auch die Dominanz der Websites mit teils konfligierenden Sachtexten als typisches Genre (Barzilai et al. 2018, 990; Primor und Katzir 2018, 5) ist ein weiteres schwierigkeitsgenerierendes Merkmal, weil die verstehensnötigen Inferenzen in Sachtexten als anspruchsvoller gelten - und zwar bereits in intratextuellen Verstehensprozessen (Lorch 2015). Dieser Befund zur Anzahl der Dokumente, dem aufgrund der teils mangelnden Daten in den Primärstudien qualitative Analysen folgen sollten, um einen blinden Fleck der Forschung auszuleuchten, verdient aus lesedidaktischer Sicht gesonderte Aufmerksamkeit.

Schliesslich ist noch anzumerken, dass das Schreiben in digitalen Settings unterbelichtet ist. Das verwundert angesichts durchaus einschlägiger Bemühungen, dem Lesen sinnvolle schriftliche Syntheseleistungen folgen zu lassen (van Ockenburg, van Weijen und Rijlaarsdam 2019), wobei sich argumentative und synoptische Texte über Gelesenes als besonders lernwirksam erweisen (Philipp 2019a, 242-50). Gerade vor dem Hintergrund, dass einerseits das Schreiben mit digitalen Medien die Schreibkompetenz auf relativ niederschwellige Art verbessert und dass andererseits analytische Schreibsettings Leseverstehensleistungen stark steigern können (Graham und Harris 2018, 21f.), lassen sich bestehende Fördermassnahmen anreichern. 
Abschliessend lässt sich damit festhalten: Diese Sekundäranalyse hat die Profile der Förderung des Leseverstehens multipler Dokumente mit und ohne Einsatz digitaler Medien am Beispiel ausgesucht effektiver Förderansätze zu konturieren versucht. Das Hauptergebnis ist, dass sich mehr Gemeinsamkeiten als Unterschiede finden liessen. Die Differenzen betrafen vor allem instruktionale Merkmale und die Materialien, mittels derer die beiden Hauptprozesse des Leseverstehens (Integrieren und Sourcing) gezielt gefördert wurden. Weniger markant sind die Unterschiede in den informatorischen Bezugspunkten der beiden Prozesse. Dass es mehr Gemeinsamkeiten als Unterschiede gibt, ist insofern positiv wertbar, als nicht grundsätzlich eine neue Lesedidaktik entwickelt werden muss. Vielmehr scheint es, als würde sich in der kontinuierlichen und nötigen Weiterentwicklung der Förderansätze ein fruchtbares Zukunftsszenario für eine Lesedidaktik 4.0 ergeben.

\section{Literatur}

Barzilai, Sarit, Asnat R. Zohar, und Shiri Mor-Hagani. 2018. «Promoting Integration of Multiple Texts: A Review of Instructional Approaches and Practices». Educational Psychology Review 30 (3): 973-99. https://doi.org/10.1007/s10648018-9436-8.

Brante, Eva Wennås, und Helge I. Strømsø. 2018. «Sourcing in Text Comprehension: A Review of Interventions Targeting Sourcing Skills». Educational PsychologY Review 30 (3): 773-99. https://doi.org/10.1007/s10648-017-9421-7.

Bråten, Ivar, Jason L. G. Braasch, und Ladislao Salmerón. 2020. «Reading Multiple and Non-Traditional Texts: New Opportunities and New Challenges». In Handbook of Reading Research, Volume V., herausgegeben von Elizabeth Birr Moje, Peter Afflerbach, Patricia Enciso, und Nonie K Lesaux. New York: Routledge. https://doi.org/10.4324/9781315676302-5.

Britt, M. Anne, und Jean-François Rouet. 2012. «Learning with Multiple Documents: Component Skills and Their Acquisition». In Enhancing the Quality of Learning: Dispositions, Instruction, and Learning Processes, herausgegeben von John R. Kirby und Michael J. Lawson, 276-314. Cambridge: Cambridge University Press.

Cheung, Alan C., und Robert E. Slavin. 2013. «Effects of Educational Technology Applications on Reading Outcomes for Struggling Readers: A Best-Evidence Synthesis». Reading Research Quarterly 48 (3), 277-99. https://doi.org/10.1002/ rrq.50. 
Cho, Byeong-Young, und Peter Afflerbach. 2017. «An Evolving Perspective of Constructively Responsive Reading Comprehension Strategies in Multilayered Digital Text Environments». In Handbook of Research on Reading Comprehension, herausgegeben von Susan E. Israel. 2. Aufl., 109-34. New York: Routledge.

Cho, Byeong-Young, Peter Afflerbach, und Hyeju Han. 2018. «Strategic Processing in Accessing, Comprehending, and Using Multiple Sources Online». In Handbook of Multiple Source Use, herausgegeben von Jason L. G. Braasch, Ivar Bråten und Matthew T. McCrudden, 133-50. New York: Routledge.

Clinton, Virginia. 2019. «Reading from Paper Compared to Screens: A Systematic Review and Meta-Analysis». Journal of Research in Reading 42 (2): 288-325. https://doi.org/10.1111/1467-9817.12269.

Delgado, Pablo, Cristina Vargas, Rakefet Ackerman, und Ladislao Salmerón. 2018. «Don't Throw Away Your Printed Books: A Meta-Analysis on the Effects of Reading Media on Reading Comprehension». Educational Research Review 25 (November): 23-38. https://doi.org/10.1016/j.edurev.2018.09.003.

Graham, Steve, und Karen R. Harris. 2018. «Evidence-Based Writing Practices: A Meta-Analysis of Existing Meta-Analyses». In Design Principles for Teaching Effective Writing: Theoretical and Empirical Grounded Principles, herausgegeben von Raquel Fidalgo, Karen R. Harris und Martine A. Braaksma, 13-37. Leiden: Brill.

Groeben, Norbert. 2002. «Dimensionen der Medienkompetenz: Deskriptive und normative Aspekte». In Medienkompetenz: Voraussetzungen, Dimensionen, Funktionen, herausgegeben von Norbert Groeben und Bettina Hurrelmann, 160-97. Weinheim: Juventa.

Kong, Yiren, Young Sik Seo, und Ling Zhai. 2018. "Comparison of Reading Performance on Screen and on Paper: A Meta-Analysis». Computers \& Education 123 (August): 138-49. https://doi.org/10.1016/j.compedu.2018.05.005.

Leu, Donald J., Charles K. Kinzer, Julie Coiro, Jill Castek, und Laurie A. Henry. 2017. «New Literacies: A Dual-Level Theory of the Changing Nature of Literacy, Instruction, and Assessment». Journal of Education 197 (2): 1-18. https://doi. org/10.1177/002205741719700202.

List, Alexandra. 2020. "Six Questions Regarding Strategy Use When Learning from Multiple Texts». In Handbook of Strategies and Strategic Processing, herausgegeben von Daniel L. Dinsmore, Luke K. Fryer, und Meghan M. Parkinson, 1. Aufl., 119-40. Routledge. https://doi.org/10.4324/9780429423635-8.

Lorch, Robert F. 2015. «What about Expository Text?» In Inferences during Reading. Hrsg. von Edward J. O'Brien, Anne E. Cook und Robert F. Lorch, 348-61. Cambridge: Cambridge University Press.

Ma, Wenting, Olusola O. Adesope, John C. Nesbit, und Qing Liu. 2014. «Intelligent Tutoring Systems and Learning Outcomes: A Meta-Analysis». Journal of Educational Psychology 106 (4): 901-18. https://doi.org/10.1037/a0037123. 
Magliano, Joseph P., Peter Hastings, Kristopher Kopp, Dylan Blaum, und Simon Hughes. 2018. "Computer-based assessment of essays based on multiple documents». In Handbook of Multiple Source Use, herausgegeben von Ivar Bråten, Jason Braasch, und Matt McCrudden, 502-26. New York: Routledge.

Magliano, Joseph P., Matthew T. McCrudden, Jean-François Rouet, und John P. Sabatini. 2017. «The Modern Reader: Should Changes to How We Read Affect Research and Theory?» In The Routledge Handbook of Discourse Processes, herausgegeben von Michael F. Schober, David N. Rapp und M. Anne Britt. 2. Aufl., 343-61. New York: Routledge.

OECD. 2019. PISA 2018 Ergebnisse (Band I): Was Schülerinnen und Schüler wissen und können. PISA. OECD. https://doi.org/10.1787/1da50379-de.

Perfetti, Charles A., Jean-François Rouet, und M. Anne Britt. 1999. «Toward a Theory of Documents Representation». In The Construction of Mental Representations during Reading, herausgegeben von Herre van Oostendorp und Susan R. Goldman, 99-122. Mahwah: Lawrence Erlbaum.

Philipp, Maik. 2018a. Lesekompetenz bei multiplen Texten: Grundlagen, Prozesse, Didaktik. Tübingen: Francke.

Philipp, Maik. «Multiple Modelle des Leseverstehens multipler Texte: Eine Synopse aktueller kognitiver Modellierungen aus lesedidaktischer Perspektive». https://www.leseforum.ch/sysModules/obxLeseforum/Artikel/646/2018_3_ de_philipp.pdf. 2018b.

Philipp, Maik. 2019a. Multiple Dokumente verstehen: Theoretische und empirische Perspektiven auf Prozesse und Produkte des Lesens mehrerer Dokumente. Weinheim: Beltz Juventa.

Philipp, Maik. 2019b. «Multiple Wege führen nach Rom: Ergebnisse einer quantitativen Sekundäranalyse effektiver Fördermaßnahmen zur Verbesserung der Sourcing- und Integrationsprozesse in der Nutzung multipler Texte». https:// www.leseforum.ch/sysModules/obxLeseforum/Artikel/665/2019_1_de_philipp.pdf.

Primor, Liron, und Tami Katzir. 2018. «Measuring Multiple Text Integration: A Review》. Frontiers in Psychology 9 (November): 2294. https://doi.org/10.3389/ fpsyg.2018.02294.

Rouet, Jean-François, und M. Anne Britt. 2014. «Multimedia Learning from Multiple Documents». In The Cambridge Handbook of Multimedia Learning, herausgegeben von Richard E. Mayer. 2. Aufl., 813-41. New York: Cambridge University Press.

Salmerón, Ladislao, Helge I. Strømsø, Yvonne Kammerer, Marc Stadtler, und Paul van den Broek. 2018. "Comprehension Processes in Digital Reading». In Learning to Read in a Digital World, herausgegeben von Mirit Barzillai, Jenny Thomson, Sascha Schroeder, und Paul van den Broek, 17:91-120. Studies in Written Language and Literacy. Amsterdam: John Benjamins Publishing Company. https://doi.org/10.1075/swll.17.04sal. 
Singer, Lauren M., und Patricia A. Alexander. 2017. «Reading on Paper and Digitally: What the Past Decades of Empirical Research Reveal». Review of Educational Research 87 (6): 1007-41. https://doi.org/10.3102/0034654317722961.

Steenbergen-Hu, Saiying, und Harris Cooper. 2014. «A Meta-Analysis of the Effectiveness of Intelligent Tutoring Systems on College Students' Academic Learning». Journal of Educational Psychology 106 (2): 331-47. https://doi.org/10.1037/ a0034752.

Swart, Elise K., Thijs M. J. Nielen, und Maria T. Sikkema-de Jong. 2019. «Supporting Learning from Text: A Meta-Analysis on the Timing and Content of Effective Feedback». Educational Research Review 28 (November): 100296. https://doi.org/10.1016/j.edurev.2019.100296.

Van Ockenburg, L., D. Van Weijen, und G. Rijlaarsdam. 2019. «Learning to Write Synthesis Texts in Secondary Education: A Review of Intervention Studies». Journal of Writing Research 10 (3): 401-28. https://doi.org/10.17239/jowr2019.10.03.01.

Salmerón, Ladislao, Helge I. Strømsø, Yvonne Kammerer, Marc Stadtler, und Paul van den Broek. 2018. "Comprehension Processes in Digital Reading». In Learning to Read in a Digital World, herausgegeben von Mirit Barzillai, Jenny Thomson, Sascha Schroeder, und Paul van den Broek, 17:91-120. Studies in Written Language and Literacy. Amsterdam: John Benjamins Publishing Company. https://doi.org/10.1075/swll.17.04sal.

$\mathrm{Xu}$, Zhihong, Kausalai (Kay) Wijekumar, Gilbert Ramirez, Xueyan Hu, und Robin Irey. 2019. "The Effectiveness of Intelligent Tutoring Systems on K-12 Students' Reading Comprehension: A Meta-Analysis». British Journal of Educational Technology 50 (6): 3119-37. https://doi.org/10.1111/bjet.12758. 